\title{
Special issue on "optimization and optimal control with applications" for the 9th international conference on optimization: techniques and applications (9th ICOTA), December 12-16, 2013, Taipei, Taiwan
}

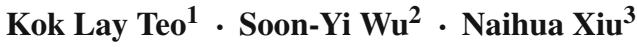

Received: 13 June 2015 / Accepted: 17 June 2015 / Published online: 11 July 2015

C Springer Science+Business Media New York 2015

\section{Preface}

Optimization is arguably one of the most useful research disciplines. It is indispensable in solving practical problems arising in many real world applications such as traditional science and engineering, natural and human resources management, economics and financial engineering.

This Special Issue is a collection of 15 full-length papers reflecting the recent advances in theory and applications of optimization from amongst the conference presentations at the 9th International Conference on Optimization: Techniques and Applications (9th ICOTA), December 12-16, 2013, Taipei, Taiwan.

This Special Issue contains 15 original research papers covering a wide range of topics in optimization and its applications. A rigorous refereeing process involving at least two referees was arranged for each of these papers to guarantee that its quality was compatible with that of a regular paper of the journal.

We wish to express our sincere appreciation to all those who have contributed to the completion of this Special Issue. In particular, we are deeply grateful to our referees who provided prompt and extensive reviews for all the submitted papers. Their valuable constructive comments have contributed to the quality of this Special Issue. We also wish to thank the Editor-in-Chief, Professor Sergiy Butenko for his kind cooperation and professional support. Our special thanks go to Mrs. Lisa Holling, Teo's Secretary, for her help during the editing process of the Special Issue. Last but not least, we would like to thank the authors of this Special Issue for their patience.

Guest Editors

\footnotetext{
Naihua Xiu

nhxiu@bjtu.edu.cn

1 Department of Mathematics and Statistics, Curtin University, Bentley, Australia

2 Department of Mathematics, National Cheng Kung University, Tainan, Taiwan

3 School of Science, Beijing Jiaotong University, Beijing, China
} 